\title{
Overcoming Noise in Entanglement Distribution
}

\author{
Sebastian Ecker®, ${ }^{1,2, *}$ Frédéric Bouchard $\odot,{ }^{3}$ Lukas Bulla, ${ }^{1,2}$ Florian Brandt, ${ }^{1,2}$ Oskar Kohout, ${ }^{1,2}$ Fabian Steinlechner, ${ }^{1,2,4,5}$

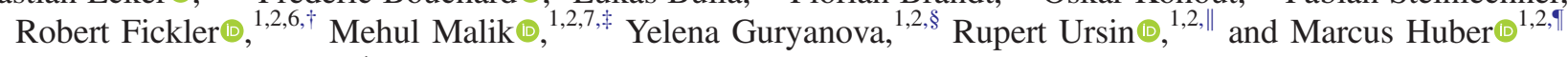 \\ ${ }^{1}$ Institute for Quantum Optics and Quantum Information (IQOQI), \\ Austrian Academy of Sciences, Boltzmanngasse 3, 1090 Vienna, Austria \\ ${ }^{2}$ Vienna Center for Quantum Science and Technology (VCQ), Faculty of Physics, \\ University of Vienna, Boltzmanngasse 5, 1090 Vienna, Austria \\ ${ }^{3}$ Department of physics, University of Ottawa, Advanced Research Complex, \\ 25 Templeton, Ottawa, Ontario, Canada, K1N 6N5 \\ ${ }^{4}$ Fraunhofer Institute for Applied Optics and Precision Engineering IOF, \\ Albert-Einstein-Strasse 7, 07745 Jena, Germany \\ ${ }^{5}$ Abbe Center of Photonics-Friedrich-Schiller-University Jena, \\ Albert-Einstein-Strasse 6, 07745 Jena, Germany \\ ${ }^{6}$ Photonics Laboratory, Physics Unit, Tampere University, Tampere, FI-33720, Finland \\ ${ }^{7}$ Institute of Photonic and Quantum Sciences (IPaQS), Heriot-Watt University, Edinburgh, Scotland, \\ United Kingdom EH14 4AS
}

(Received 1 August 2019; revised manuscript received 11 October 2019; published 26 November 2019)

Noise can be considered the natural enemy of quantum information. An often implied benefit of highdimensional entanglement is its increased resilience to noise. However, manifesting this potential in an experimentally meaningful fashion is challenging and has never been done before. In infinite dimensional spaces, discretization is inevitable and renders the effective dimension of quantum states a tunable parameter. Owing to advances in experimental techniques and theoretical tools, we demonstrate an increased resistance to noise by identifying two pathways to exploit high-dimensional entangled states. Our study is based on two separate experiments utilizing canonical spatiotemporal properties of entangled photon pairs. Following these different pathways to noise resilience, we are able to certify entanglement in the photonic orbital-angular-momentum and energy-time degrees of freedom up to noise conditions corresponding to a noise fraction of $72 \%$ and $92 \%$, respectively. Our work paves the way toward practical quantum communication systems that are able to surpass current noise and distance limitations, while not compromising on potential device independence.

DOI: 10.1103/PhysRevX.9.041042

Subject Areas: Photonics,

Quantum Physics,

Quantum Information

\section{INTRODUCTION}

Quantum entanglement is one of the most peculiar and elusive properties of quantum systems, a key resource in quantum information processing [1], and an indispensable

\footnotetext{
*sebastian.ecker@oeaw.ac.at

†robert.fickler@tuni.fi

${ }^{\star}$ m.malik@hw.ac.uk

§yelena.guryanova@oeaw.ac.at

rupert.ursin@oeaw.ac.at

"marcus.huber@univie.ac.at
}

Published by the American Physical Society under the terms of the Creative Commons Attribution 4.0 International license. Further distribution of this work must maintain attribution to the author(s) and the published article's title, journal citation, and DOI. ingredient for device-independent quantum cryptography [2]. At the same time, entangled quantum systems are highly delicate since their entanglement is readily diminished by the slightest interaction with the environment. This is of particular relevance for the distribution of entangled photons over long distances outside of a protected laboratory environment, where particle loss and environmental noise are inevitable. Similar to classical communication, noise ultimately reduces the channel capacity and thus acts as a limiting factor for the link distance in quantum communications. Several proof-of-concept experiments have pushed the distribution distance of two-dimensional-entangled photon pairs over fiber [3-5] and free-space [6-8] links, while others have demonstrated the distribution of highdimensional entangled quantum states [9-14].

Although it is not straightforward to certify highdimensional entanglement from experimental data, its 
production in the process of spontaneous parametric down-conversion (SPDC) happens naturally. As a result of conservation laws in this process, the down-converted photon pairs are entangled in spatiotemporal properties such as energy-time [15-18], angle-angular momentum [19-22], and position-momentum [23-25].

At first glance, from an abstract information theoretic point of view, high-dimensional entanglement might seem to be essentially reproducible by just many copies of regular qubit entanglement. While there is actually a notable difference even in idealized pure states [26] and cryptographic settings [27], one of the main reasons for developing high-dimensional protocols has predominantly been the aforementioned free availability in downconversion combined with the capability of storing more bits per communicated photon. Indeed, many such benefits of using high-dimensional encodings in quantum key distribution (QKD) have been investigated in the past decade [28-32], followed by experimental implementations in recent years [33-37]. Apart from an increased perphoton information capacity, an often implied advantage of employing high-dimensional entanglement is its potential for increased resistance to noise.

While it is indeed true that dimension-independent noise models show an increased resistance of entangled states to noise $[38,39]$, the actual advantages very much depend on the physical implementation. Different highdimensional degrees of freedom (d.o.f.) are bounded by different operational constraints. Thus, it has remained an open question whether practical improvements using highdimensional entanglement can actually manifest its promised advantages.

In this paper, we expound potential pathways to an increased resilience to noise by utilizing entanglement in high dimensions. We conduct two experiments, exploiting the most paradigmatic platforms for generating highdimensional entangled quantum states, namely photons entangled in energy-time as well as transverse positionmomentum. We show that for each high-dimensional encoding method and its associated state-of-the-art technology, there is an appropriate pathway to verify entanglement in conditions where qubit entanglement cannot be distributed due to extreme external noise levels. We are further able to characterize a realistic trade-off between dimensionality and robustness to find optimal and flexible encodings for both implementations and different background conditions, thereby revealing the transformative potential of high-dimensional quantum information.

\section{PATHWAYS TO NOISE RESILIENCE}

Almost all quantum experiments aim to harness a physical process that is expected to yield a pure entangled state. If the system is bipartite, and assuming that the experiment is ideal, then the entangled state can be represented in the Schmidt basis $\left|\psi_{A B}\right\rangle=\sum_{i} \lambda_{i}|i i\rangle$.
Needless to say, experiments are seldom ideal, and a number of factors contribute to spoiling the state, during both its generation and its manipulation. Errors could, for example, be introduced during the distribution of the state via quantum channels or through imperfect measurement devices. Moreover, background photons inevitably introduce noise, resulting in a reduction of the signal-to-noise ratio at the readout. It is well known that noise deteriorates entanglement, and the extent to which entanglement persists despite the presence of noise is known as "noise resistance of entanglement" [1]. The degree to which the initially pure state is degraded is often estimated using a white noise model, i.e., by mixing the target state $|\psi\rangle$ with the maximally mixed state:

$$
\hat{\rho}=p|\psi\rangle\langle\psi|+\frac{1-p}{d^{2}} \mathbb{1}_{d^{2}} .
$$

One may also note that this model captures particle loss for the maximally entangled state $|\psi\rangle=\left|\Phi^{+}\right\rangle:=$ $(1 / \sqrt{d}) \sum_{i}|i i\rangle$, where with probability $p$ the state remains intact, and with probability $1-p$ a particle from a pair is lost. The measurement statistics of the lost photon correspond to the maximally mixed state, while the statistics of the partner photon are replaced by the marginal. In the case of the maximally entangled state, this marginal is also maximally mixed, $\operatorname{Tr}_{B}\left|\Phi^{+}\right\rangle\left\langle\Phi^{+}\right|=(1 / d) \mathbb{1}$, resulting in the model in Eq. (1). For this "isotropic" state, the resulting tolerance to noise, i.e., the critical $p_{c}$ after which the state becomes separable, scales as $p_{c}=[1 /(d+1)]$. This can already be concluded from the first criteria for mixed state entanglement, such as positivity under partial transposition $[40,41]$, and has already been pointed out in early literature $[42,43]$. While for general states such resistance to depolarizing noise is quite generic [44], physical modeling can reveal even further avenues of avoiding noise in high dimensions [38]. We argue in Sec. III, that the noise introduced in both our experiments is close to white. Nonetheless, it is important to emphasize here that we do not assume any noise model when analyzing the experimental data for entanglement-the simple noise model serves only as a motivating example for why we should be expecting an increased noise resistance and it is not needed for performing or analyzing the experiment. In a realistic experimental setting, loss can affect the measurement statistics in more complex ways, such as introducing accidental coincidence counts due to detector or background noise. A more quantitative analysis of the precise role of noise in photonic entanglement has been performed recently [38] and supports our experimental results by demonstrating a clear advantage of going to high dimensions. For more general states, bipartite depolarizing maps [44] capture different loss rates or detection efficiencies and can be solved analytically for any dimension. The common feature of these noise models is the fact that it is possible for 
the noise resistance to increase linearly with the system dimension $d_{S}$. As $d_{S}$ grows, so does the so-called "dimensionality of entanglement." Thus, one should, in principle, be able to overcome any amount of noise, and detect entanglement, simply by looking in systems of high enough dimension. Despite this feature, there are several reasons why this idea has not yet manifested in any practical setups. First, certifying entanglement requires one to collect enough information about the underlying quantum state. The number of measurements required to do this scales at least linearly with the size of the system [45]. Second, the dimension of a system is not a fundamentally tunable parameter in an experiment. Finally, the noise very much depends on the physical implementation of the chosen scheme. The theoretical description of spatiotemporal d.o.f. of any photon state is infinite dimensional,

$$
|\psi\rangle_{\text {photon }}=\sum_{s} \int d \mu(x) \psi_{s}(x)|x, s\rangle
$$

where $s$ is the polarization d.o.f., $x$ is the position, and $\mu(x)$ is a measure over the space. It then follows that the description of temporally or spatially entangled photon states is also infinite dimensional. Despite this, any laboratory measurement still requires one to discretize these d.o.f. The discretization depends on the measuring device; for example, to discretize temporally entangled states one could time resolve photon detections using high-precision clocks.
For states entangled spatially, one could perform spatial mode measurements using spatial light modulators or cameras. All of these techniques have limited resolution; this means that by increasing the dimension of the states (i.e., discretizing further), one will often encounter additional sources of noise, e.g., through cross talk or additional measurement channels, which consequently lead to dimension-dependent noise factors $p(d)$ entering into the models. Thus, while high-dimensional entanglement presents an increased resistance to noise with increasing dimension on paper, it is not clear whether this theoretical advantage can be exploited in a real experiment.

Nevertheless, noise resistance of entanglement is a highly desirable feature in quantum communication and is of utmost importance for fundamental reasons. If one is able to demonstrate the persistence of entanglement, simply by discretizing the description of systems, then one may be closer to understanding the fundamental limits on the information capacity of single-photon quantum communication channels. In spite of this potential, not a single quantum experiment to date has been able to show an increase in noise resistance in a controlled fashion. In this work, we present two experiments that discretize continuous d.o.f. to encode information in high-dimensional quantum systems to explicitly demonstrate an increased resilience to environmental noise. These paradigms are illustrated in Fig. 1. It is to be read as a flow chart, starting in the center where a hypothetical noisy quantum state $\rho$ is

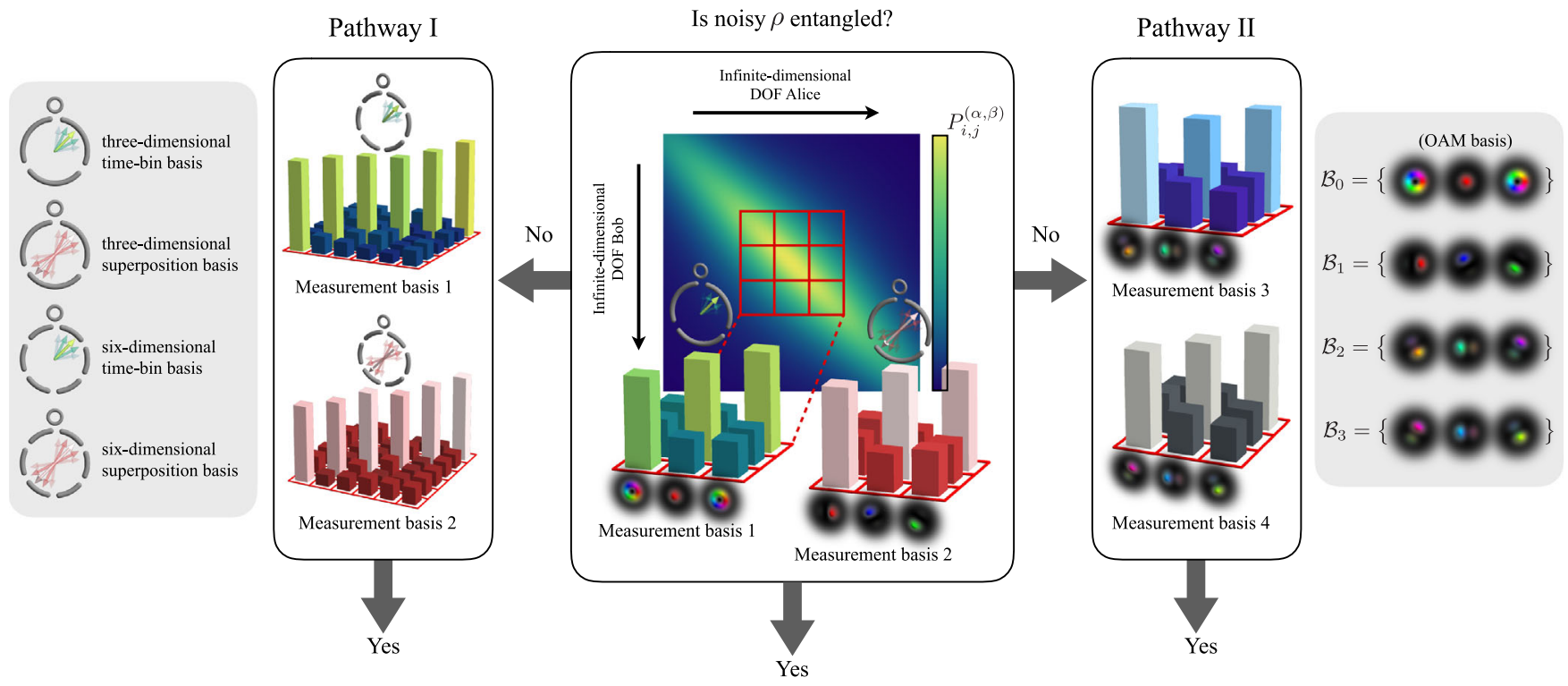

FIG. 1. Illustration of the pathways to noise resilience. A mixed entangled state $\rho$ shared by Alice and Bob is encoded in spatiotemporal properties of photon pairs. Each of the infinite-dimensional d.o.f. of the photons can be discretized and measured in two bases (center panel). If both measurements are insufficient to certify entanglement in the noisy state, there are two pathways to recover it: fine-graining to higher dimensions (pathway I, left-hand panel) and measuring in additional bases (pathway II, right-hand panel). In pathway I, noise is "diluted" by discretizing the existing state space further, resulting in an increased signal-to-noise ratio. Pathway II exploits the existence of more than two mutually unbiased measurement bases in higher dimensions, providing additional information about the nonclassicality of the state. The bar charts illustrate the joint probability $P_{i, j}^{(\alpha, \beta)}$ of measuring Alice's modes $i$ in the basis $\alpha$ and Bob's modes $j$ in the basis $\beta$. 
tested for entanglement by making measurements in two bases. If none is found, one has two options, depending on the d.o.f. and its technological constraints. The first pathway (Fig. 1, left) is to fine-grain or partition the quantum state to higher dimensions, for example, by discretizing an energy-time entangled state to a higher temporal resolution. Alternatively, one may explore pathway II (Fig. 1, right), which exploits the existence of more than two mutually unbiased bases (MUBs) in high dimensions. For example, measurements of an orbital-angular-momentum (OAM) entangled state can be made in additional bases, providing more information about the state. In both cases, entanglement can be recovered from a state in an assumption-free manner, where no entanglement could previously be certified through standard techniques.

\section{EXPERIMENTAL IMPLEMENTATION}

Here, we showcase two photonic experiments that demonstrate high-dimensional noise resilience of entanglement via the above-described two pathways. In the first experiment we follow pathway I and exploit energy-time entanglement, while in the second experiment we take pathway II to explore the orbital-angular-momentum d.o.f., both encodings that have seen rapid experimental progress in recent years [1]. The basic premise of both experiments is to create photon pairs and show, via a set of appropriately chosen measurements, that these pairs remain entangled even in the presence of high levels of noise. To generate the pairs in both experiments we appeal to spontaneous parametric down-conversion.

First, let us consider the creation of photon pairs entangled in energy-time. In the nonlinear SPDC process, a crystal pumped with photons of frequency $\omega_{P}$ will spontaneously produce a pair of photons with frequencies $\omega_{0}$ and $\omega_{1}$. The total energy is strictly conserved such that, despite the crystal producing photon pairs with a finite bandwidth, the sum of their frequencies is constant: $\hbar \omega_{P}=\hbar \omega_{0}+\hbar \omega_{1}$. This results in the emission of two photons that are highly entangled in energy. Since the spectral linewidth and the coherence time are inversely related, a narrow pump bandwidth results in a long coherence time for possible photon pair emissions, giving rise to entanglement in the time domain with Schmidt numbers up to $\sim 10^{9}$ under realistic experimental assumptions $[33,46]$. In our scheme we utilize ancillary entanglement in the polarization d.o.f. to facilitate interference in the time domain.

A similar narrative holds for the second experiment, which produces photons entangled in the orbital-angularmomentum d.o.f. Here, the strict conservation of momentum in the SPDC process, $\hbar l_{p}=\hbar l_{0}+\hbar l_{1}$, results in the production of photon pairs anticorrelated in OAM, $\hbar l_{0}=$ $-\hbar l_{1}$, for a Gaussian-mode pump photon with $\hbar l_{p}=0$, leading to entanglement in the OAM-angular position variables [21]. The (theoretically) infinite-dimensional states produced by the two experiments can be written as

$$
\begin{gathered}
|\Psi\rangle_{\mathrm{ET} \text {-pol }}=\int d t f(t)|t\rangle_{A}|t\rangle_{B} \otimes\left|\phi^{-}\right\rangle_{A B}, \\
|\Psi\rangle_{\mathrm{OAM}}=\sum_{\ell=-\infty}^{\infty} c_{\ell}|-\ell\rangle_{A}|\ell\rangle_{B},
\end{gathered}
$$

where $f(t)$ is a continuous function of time, corresponding to the coherence profile of the laser, $\left|\phi^{-}\right\rangle_{A B}=$ $(1 / \sqrt{2})\left(|H\rangle_{A}|H\rangle_{B}-|V\rangle_{A}|V\rangle_{B}\right)$ is a polarization-entangled Bell state, $| \pm \ell\rangle$ is the state of a photon carrying an OAM quantum number of $\pm \ell$, and $c_{\ell}$ is a complex probability amplitude, which is defined by the spatial characteristics of the crystal and pump beam.

In order to gain meaningful insight into noise resilience, both states must be appropriately discretized. In the energytime experiment, we measure the time of arrival of entangled photon pairs by discretizing a time frame of duration $F$ into bins and recording which bin a photon is detected in. The duration of $F$ is fixed and we divide it into an integer number of time-bin modes $d$, each corresponding to a duration $t_{d}$, i.e., $F / d=t_{d}$ (see Supplemental Material, Sec. III [47]). In pathway II, we choose a finite cutoff to the theoretically infinite sum over modes, such that the modes with OAM quantum numbers $l \in\{-D, \ldots, D\}$ are spanning a $(2 D+1)$-dimensional Hilbert space. Thus, ideally, the states generated by the experiments would be close to the forms

$$
\begin{gathered}
|\Psi\rangle_{\mathrm{ET}-\mathrm{pol}}=\sum_{j=1}^{d} \alpha_{j}|j\rangle_{A}|j\rangle_{B} \otimes\left|\phi^{-}\right\rangle_{A B}, \\
|\Psi\rangle_{\mathrm{OAM}}=\sum_{\ell=-D}^{D} c_{\ell}|-\ell\rangle_{A}|\ell\rangle_{B},
\end{gathered}
$$

where $|j\rangle$ refers to a photon in a discrete time-bin state whose duration is $t_{d}$ for $j \in\{1, \ldots, d\}$ and $\alpha_{j}$ is a complex probability amplitude.

Despite investigating different d.o.f., the experiments have similar characteristic features, as shown in Fig. 2. In both schemes, a nonlinear crystal is pumped with a continuous-wave diode laser to generate photon pairs, which then pass through a setup consisting of measurement elements and an external noise source. In addition, the entanglement dimensionality for both cases ( $d$ for energytime and $d=2 D+1$ for OAM) is strongly dependent on the pump characteristics. In the energy-time experiment [Fig. 2(a)], a narrow-bandwidth pump ensures a large Schmidt number, while in the OAM experiment [Fig. 2(b)], a large pump mode with a well-defined transverse momentum results in high-dimensional OAM entanglement. For additional experimental details, see the Appendixes A and B. In both experiments, noise is introduced in the form of background photons generated by sources of light simulating a realistic operational environment for a quantum communication system. In the energy-time experiment, 


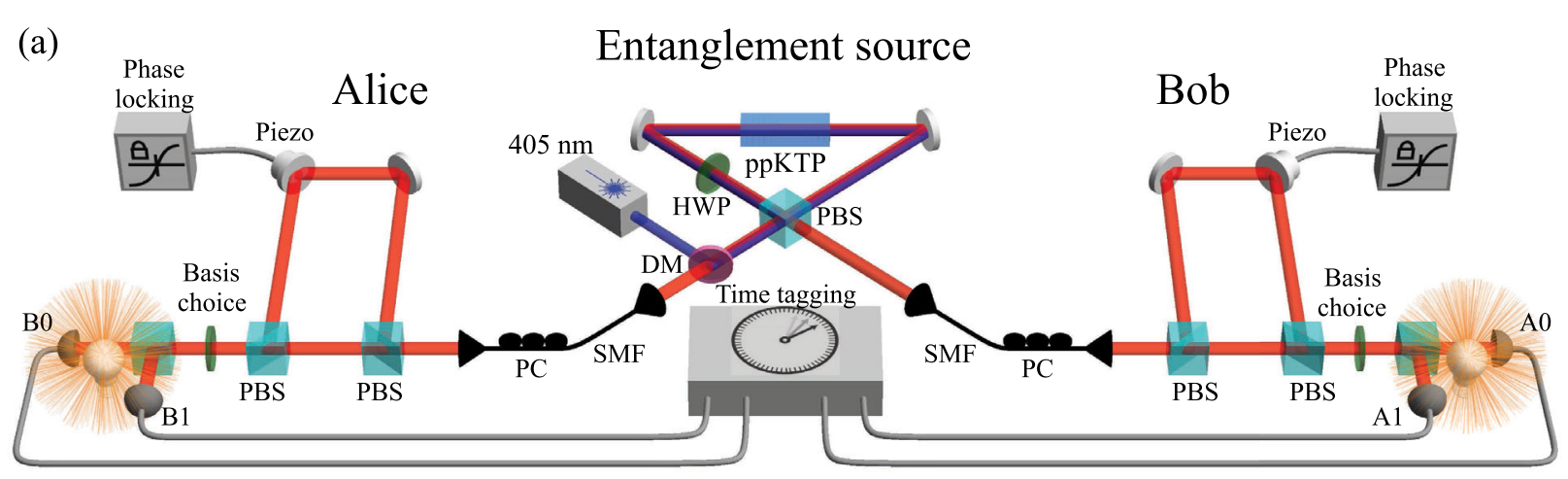

(b)

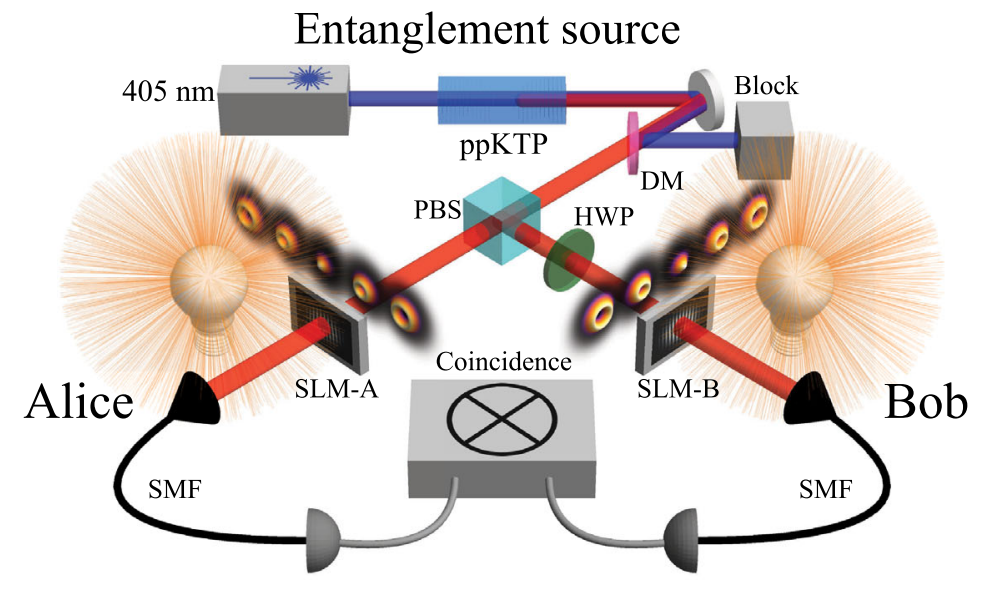

(c)

FIG. 2. Experimental setup for (a) energy-time and (b) orbital angular momentum (OAM) d.o.f. In both experiments, a 405-nm continuous-wave laser produces high-dimensionally entangled photon pairs in a ppKTP crystal exploiting type-II spontaneous parametric down-conversion (SPDC). The noise is optically added by intensity-adjustable light sources and single-photon detection is accomplished using avalanche photodiodes. (a) Additional polarization entanglement is generated by bidirectionally pumping the crystal in a polarization Sagnac interferometer. The polarization basis the photon pairs are measured in after an actively stabilized postselection-free Franson interferometer defines the measurement basis in the time domain. Each detection event is time tagged and recorded by means of a time-to-amplitude converter. (b) The OAM-entangled pairs are split depending on their polarization, analyzed through mode filtering by modulating the complex amplitude of the photons, and subsequently coupled into SMFs. Coincidence counts are recorded using a coincidence logic.

fine-adjustable light emitting diodes placed near the detectors introduce background counts-simulating a scenario where classical light may be copropagating with a quantum signal. In the OAM experiment, background counts are introduced by increasing the intensity of the ambient light in the lab up to daylight conditions, which is a realistic scenario for freespace experiments using large aperture telescopes. White noise is generated in the energy-time experiment by employing two independent noise sources for Alice and Bob, thus eliminating temporal correlations, while in the OAM experiment white noise is introduced by placing the noise source after the spatial light modulators, ensuring mode-independent noise generation. In both cases, we quantify the amount of noise introduced via the noise fraction NF, which corresponds to the fraction of counts in our data that arise from noise. Intuitively, $\quad \mathrm{NF}=[($ no. noise counts $) /($ no. total counts $)]$, which takes on values from 0 (no noise) to 1 (complete noise). A more rigorous definition of the noise fraction NF and its computation from experimental data is presented in the Supplemental Material, Sec. II [47].

\section{ENERGY-TIME ENTANGLEMENT (PATHWAY I)}

The first pathway to noise resilience is implemented by fine-graining measurements of the photon arrival time. As outlined, we discretize a time frame into $d$ time bins and record the bin that a photon is detected in. The goal of the experiment is simple: by increasing the dimension $d$ of the state in Eq. (5) through fine-graining, we want to certify entanglement of noisy quantum states, which is otherwise concealed by noise.

To this end, we collect statistics about the state in two bases. The first measurement is in the same basis as the state in Eq. (5). Projecting onto the time-bin states $|i, j\rangle$, with $i, j \in\{1, \ldots, d\}$, is accomplished by recording the 
time of arrival of single photons with a detector and a precise clock, which constitutes a multioutcome measurement. The second measurement is more difficult, as it must be performed in a superposition basis of the time-bin states. This can be achieved by delaying the state $|i\rangle$ for a duration corresponding to $f$ time bins and subsequently interfering it with the state $|i+f\rangle$. We realize this in our experiment by utilizing a Franson interferometer [49], which employs an unbalanced interferometer for Alice and Bob, respectively [see Fig. 2(a)]. The long interferometer arm delays the state $|i\rangle$ relative to the state $|i+f\rangle$, which occupies the spatial path of the short interferometer arm. The second basis therefore projects onto the states $(1 / \sqrt{2})\left(|i, j\rangle+e^{i \phi} \mid i+\right.$ $f, j+f\rangle)$, where the phase $\phi$ is set by the sum of the two individual interferometer phases and $i, j \in\{1, \ldots, d\}$. However, without active switching, this interferometer will also project onto the states $|i, i+f\rangle$ and $|i+f, i\rangle$, which are not interfering and thus must be discarded in coincidence postselection. Since we investigate high-dimensional states, these noninterfering events are part of our state space and we may not simply discard them. We tackle this problem by employing a postselection-free Franson interferometer [50]. In this scheme, polarization entanglement is exploited to deterministically route the photon pairs in the Franson interferometer. This requires a hyperentangled source state [51,52] in polarization and energy-time. We generate the additional polarization entanglement by bidirectionally pumping a nonlinear crystal centered in a polarization Sagnac interferometer [53,54], which enables

(a)

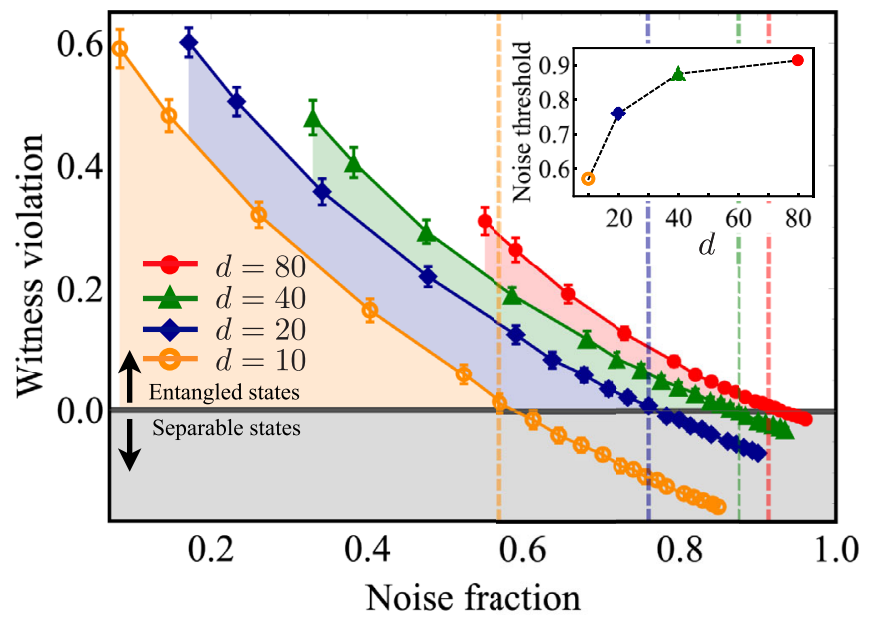

us to use the polarization d.o.f. to switch between the two measurement basis in the time domain (see Appendix A for details). Our entanglement certification is based on a dimension-dependent entanglement witness $W\left(\rho_{\mathrm{ET}}, d\right)$, where, from Eq. (5), $\rho_{\mathrm{ET}}=\operatorname{Tr}_{\mathrm{pol}}|\Psi\rangle\langle\Psi|$. The relation from the count statistics of the two measurements to the state $\rho_{\mathrm{ET}}$ is rather involved and can be found in the Supplemental Material, Sec. I [47]. Here, it suffices to say that our state is entangled if $W\left(\rho_{\mathrm{ET}}, d\right)>0$.

We introduce increasing levels of external noise corresponding to a noise fraction NF ranging from 0 to nearunity, in order to transition from a close-to-pure to a mixed state. Following pathway I, we now fine-grain our state space to higher dimensions. The frame duration $F$ is fixed at 320 clock cycles and we discretize the frame in four ways according to $F / d=t_{d}$ for $d \in\{10,20,40,80\}$. This choice of dimensions depends on the imbalance of the Franson interferometer, and is detailed in Appendix A. Figure 3(a) illustrates the scaling of the entanglement witness $W$ for different dimensions as the noise fraction NF is increased. This increase is accomplished by incrementing the amount of external optical noise, with the sequence of data points in each dimension corresponding to the same external noise levels. The noise threshold, which is the maximal NF for which entanglement can be certified, increases with higher dimensions, indicating noise resilience (see inset). For $d=10,20,40,80$, the noise thresholds steadily increase from $0.57,0.76,0.86$ to 0.93, respectively. As a consequence of fine-graining,

(b)

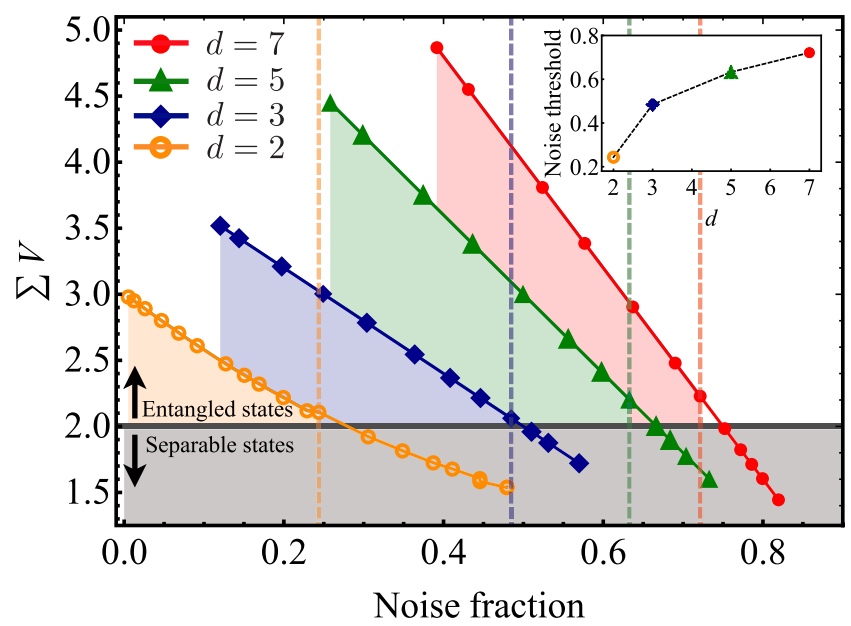

FIG. 3. Main results of our experimental demonstration of noise resistance for (a) energy-time entanglement and (b) OAM entanglement. Each plot depicts the violation strength of a suitably chosen entanglement witness against the noise fraction, i.e., the fraction of coincidence detections attributable to noise. In (a), the principal competition in achieving noise resistance is clearly visible. As the dimensionality is increased through fine-graining (pathway I), more noise is induced (and thus the curves move to the right), while a higher noise resilience is achieved (thus the noise threshold also moves to the right). Plot (b) is qualitatively different, as it explores pathway II. Instead of fine-graining, more modes are included in the analysis which allow for an increased number of mutual unbiased bases to be measured and thus also show a higher noise threshold with increasing dimension. The error bars correspond to 3 standard deviations of the mean, calculated by propagating the Poissonian error in the photon-counting rates via a Monte Carlo simulation; see Supplemental Material, Sec. IV [47]. In (b), the error bars are smaller than the data points. 
the cross talk between time bins increases due to fundamental and technical limitations. This excess noise becomes relevant once the time-bin size is smaller than the timing resolution of the detectors, as is the case with $d=40$ and $d=80$. For these discretizations, the NF is significantly increased even in the absence of external noise, indicated by the first data points in each dimension. Fine-graining at low external noise levels also reduces the witness violation, while for noise levels close to the noise threshold, fine-graining results in the recurrence of otherwise obscured entanglement.

\section{ORBITAL ANGULAR MOMENTUM ENTANGLEMENT (PATHWAY II)}

The second pathway to noise resilience takes advantage of the larger number of mutually unbiased bases in higher dimensions. Here, we explore this pathway using measurements of orbital angular momentum MUBs, for which precise measurement techniques have only recently been developed [55]. Mutually unbiased bases are an invaluable tool in many quantum information tasks, such as quantum state tomography, quantum cryptography, and entanglement certification. They consist of a set of orthonormal bases $\left\{\mathcal{B}_{\alpha}\right\}$, where $\mathcal{B}_{\alpha}=\left\{\left|\psi_{m}^{(\alpha)}\right\rangle\right\}, m \in\{0,1, \ldots, d-1\}$, and $\alpha \in\{0,1, \ldots, d\}$. Such a set is called mutually unbiased if and only if

$$
\left|\left\langle\psi_{m}^{(\alpha)} \mid \psi_{n}^{(\beta)}\right\rangle\right|^{2}=\delta_{\alpha \beta} \delta_{m n}+\left(1-\delta_{\alpha \beta}\right) / d,
$$

where $\delta_{i, j}$ is the Kronecker delta. In dimensions that are powers of prime numbers, it is known that there exist exactly $(d+1)$ MUBs. Surprisingly, for dimensions that are not powers of prime numbers, finding the number of MUBs and their elements remains an open problem [56]. For the case of prime dimensions and $\alpha \geq 1$, a MUB element is explicitly given by $\left|\psi_{m}^{(\alpha)}\right\rangle=(1 / \sqrt{d}) \sum_{j=0}^{d-1}\left(\omega_{d}^{m}\right)^{d-j}\left(\omega_{d}^{-(\alpha-1)}\right)^{s_{j}}|j\rangle$, where $\omega_{d}=\exp (2 \pi i / d)$ and $s_{j}=j+\cdots+(d-1)$. In the current experiment, we use the intensity flattening technique [55] to measure the correlations of the photon pairs in all MUBs (see Appendix B for further details). The joint probability of Alice and Bob measuring states $\left|\psi_{m}^{(\alpha)}\right\rangle$ and $\left|\psi_{n}^{(\beta)}\right\rangle$, respectively, is given by $P^{(\alpha, \beta)}(m, n)$. For a complete set of joint measurements by Alice and Bob in bases $\mathcal{B}_{\alpha}$ and $\mathcal{B}_{\beta}$, respectively, we define the correlation visibility as $V^{(\alpha, \beta)}=\sum_{i=0}^{d-1} P^{(\alpha, \beta)}(i, i)$. Following the analysis of Ref. [57], we obtain an upper bound for separable states by considering the sum of the visibilities over $k$ MUBs; i.e., $\sum_{j}^{k-1} V^{(j, j)} \leq 1+[(k-1) / d]$. In particular, for measurements in all $k=(d+1)$ MUBs, entanglement certification is achieved for $\sum_{j}^{k-1} V^{(j, j)}>2$. Hence, in contrast to the case of energy-time entanglement described before, where detections are limited to measurements in two-dimensional subspaces but dimensions of up to 80 , we are now able to fully characterize the generated states by performing high-dimensional projective measurements, but we are limited to lower overall dimensions. However, this might be largely increased by using custom-tailored phase matching [58] or by considering the complete space of transverse spatial modes, namely, radial modes along with azimuthal modes.

As a starting point, we consider bidimensionally entangled OAM states of the form $(|1,-1\rangle+|-1,1\rangle) /$ $\sqrt{2}$. Entanglement is certified by measuring correlations in all three MUBs in the two-dimensional space of OAM $|\ell= \pm 1\rangle$. Environmental noise is steadily added by gradually increasing the intensity of the ambient light present in the lab, corresponding to a noise fraction NF ranging from 0 to 0.8 . Figure 3(b) shows how the sum of visibilities $\left(\sum V\right)$ in $d+1$ MUBs varies as a function of increasing noise fraction. Entanglement is always certified if $\sum V>2$, irrespective of dimension. For $d=2$, entanglement is certified for noise fractions up to 0.24 . However, with increasing dimension, we are able to tolerate a higher noise fraction threshold, beyond which no entanglement can be certified (see inset). For $d=3,5$, and 7, the noise fraction thresholds are $0.48,0.63$, and 0.72 , respectively. The inset also shows that the noise threshold seems to be saturating as the dimension is increased. This is primarily due to the reduced fidelity of measurements in high dimensions, as well as our state moving further away from an ideal maximally entangled state as the dimension is increased. However, it is clear from our results that by increasing the state dimension, which in turn enables measurements in more bases, one can increase the resilience of entanglement to background noise. It is interesting to note that this could motivate the search for high-dimensional MUBs for any dimension, as communication systems should ideally be able to optimally operate beyond prime dimensions.

\section{DISCUSSION}

Our experimental results showcase the challenges and potential of overcoming noise through high-dimensional entanglement in quantum communication. While the necessary spatiotemporal entanglement is routinely generated in down-conversion, the real challenge is to encode information in these high-dimensionally entangled states. In other words, high-dimensional entanglement is already present in the workhorses of quantum communication, but routinely lost through coarse-graining and ignorance of modes. While this can be beneficial in removing noise from the signals, we observe a competition between two key factors: High-dimensional encoding increases the noise resistance as the dimension grows through the two pathways we identified, but also adds additional noise with increasing dimension. This is a competition that will ultimately always be won by noise; otherwise, single photons could carry an infinite amount of information. The ultimate goal is finding the sweet spot, where the 
increased noise resistance still trumps the additional noise and thus realizes a practical improvement in noisy entanglement distribution. What we show in our two experiments is that this sweet spot is actually beyond dimension two and thus defies conventional wisdom in the field, calling for the development of high-dimensional protocols across photonic platforms. While we have used two different experiments to illustrate the two pathways to noise resistance separately, both pathways could in principle be realized simultaneously in the same experiment. If one had access to multiple MUB measurements in time bins or multioutcome measurements in the spatial domain, one could harness both pathways, leading to an increase in measurable dimensionality and, as a consequence, higher noise thresholds.

Our method of adding external noise, namely by fixing a constant luminosity light source close to our detectors, is a fairly realistic model of noise that captures the decreased signal-to-noise ratio in long-distance quantum communication, where detector dark counts start dominating the distance-attenuated single-photon pairs. On the other hand, our experiments also simulate daylight conditions for freespace quantum communication $[59,60]$, where background photons will trigger accidentals in the very same way as our artificial lamps do. In both of these scenarios, the most detrimental noise in the quantum channel is white, which motivated us to employ noise sources of this characteristic in our experiments.

The most remarkable outcome of this study is the fact that we demonstrate the possibility to certify entanglement that was otherwise obscured. In other words, entanglement really was able to overcome physical noise in the implementation and reveal itself by going to higher-dimensional encodings. We would like to note that this is not only a proof-of-principle implementation, but it is also ready to be directly adopted for long-distance or free-space quantum communication $[9,61]$. At least for the energy-time experiment, we could use the exact same setup, whereas for the OAM experiment, we would require a multioutcome measurement, such as the recently developed spatial mode sorter [62]. With the current single-outcome measurements, every element or dimension we add will experience the same environmental noise [since it directly couples to the single-mode fiber (SMF)], thus unfavorably influencing the competition between noise and entanglement, with the total noise fraction increasing at the same rate as the additional noise robustness. The noise fraction we measured nonetheless proves that if one had a measurement technique where the noise distributes over multiple channels, we would have a tremendously increased resistance to physical noise outside of laboratory settings.

The obvious next challenge is the development of quantum communication protocols that make direct use of high-dimensional encodings. The fact that entanglement can be certified under extremely noisy background conditions motivates the question of whether such noisy entanglement can indeed be used to certify security of QKD or aid in other quantum information tasks. It has recently been proven that every entangled state, no matter how noisy, provides an advantage in entanglement-assisted classical communication [63]. In addition, every noisy entangled state also provides an advantage for the task of channel discrimination [64]. We hope that this study spurs further investigation into information theoretic protocols based on high-dimensional and noisy entangled states, which can be distributed in regimes where no qubit communication is possible.

\section{ACKNOWLEDGMENTS}

We thank Jessica Bavaresco for helpful discussions on OAM entanglement certification and data analysis. F. Brandt, M. H., M. M., R. F., and Y. G. acknowledge funding from the Austrian Science Fund (FWF): Y879-N27, I3053N27, P31339-N27. M. M. acknowledges support from the QuantERA ERA-NET co-fund [Austrian Science Fund (FWF): I3773-N36] and from the UK Engineering and Physical Sciences Research Council (EPSRC) (EP/ P024114/1). F. Bouchard acknowledges the support of the Vanier Canada Graduate Scholarships Program and the Natural Sciences and Engineering Research Council of Canada (NSERC). R. F. acknowledges the support of the Academy of Finland (Competitive Funding to Strengthen University Research Profiles-decision 301820 and Photonics Research and Innovation Flagship-decision 320165). We acknowledge funding from the Austrian Research Promotion Agency (FFG) Quantenforschung und -technologie (QFTE) Contract No. 870003, Austrian Science and Applications Programme (ASAP) Contracts No. 854022 and No. 866025, and ESA European Space Agency Contract No. 4000112591/14/NL/US.

S. E. and F. Bouchard contributed equally to this work; M. M., R. U., and M.H. conceived the project; S. E., L. B., and O.K. designed and developed the energy-time entanglement experiment under the guidance of F.S. and R. U.; F. Bouchard and F. Brandt designed and developed the orbital-angular-momentum entanglement experiment under the guidance of R.F., M.M., and M.H.; M.H. and Y. G. established the entanglement certification methods; S. E., F. Bouchard, R. F., M. M., Y. G., and M.H. wrote the first draft of the manuscript; all authors discussed the results and reviewed the manuscript; $\mathrm{M}$. H. supervised the whole project.

\section{APPENDIX A: ENERGY-TIME ENTANGLEMENT EXPERIMENT}

The experimental setup can be divided into a hyperentangled photon pair source, a Franson interferometer consisting of two imbalanced polarizing Mach-Zehnder interferometers (PMZIs), and a detection and time-tagging unit. Our source is based on SPDC in a 20-mm-long 
periodically poled potassium titanyl phosphate (ppKTP) crystal designed for type-II quasi-phase-matching. A grating-stabilized photodiode (Toptica DL pro) emitting at a wavelength of $405 \mathrm{~nm}$ is generating the pump field for the SPDC. Because of a narrow pump bandwidth of $\Delta \nu_{\mathrm{FWHM}} \sim 500 \mathrm{kHz}$, the down-converted signal and idler fields are energy-time entangled within a coherence time of $t_{\text {coh }}=1 /\left(\pi \Delta \nu_{\text {FWHM }}\right) \sim 636 \mathrm{~ns}$. The ppKTP crystal is temperature tuned to produce wavelength-degenerate photon pairs at $810 \mathrm{~nm}$. In order to obtain polarization entanglement, the crystal is bidirectionally pumped in the center of a polarization Sagnac interferometer [53,54]. After 3-nm bandpass filtering and single-mode coupling, we detect an entangled photon pair rate of $15 \mathrm{kcps} / \mathrm{mW}$ of pump power with a heralding efficiency of $20 \%$ in both signal and idler modes.

The single photons are then guided to two bulk optics PMZIs with an imbalance between long and short interferometer arm of $2.67 \mathrm{~ns}$. The imbalance of the two PMZIs is matched up to the correlation length of the photon pairs $(\sim 800 \mu \mathrm{m})$. By adjusting the phases $\phi_{A / B}$ of Alice's or Bob's PMZI, we see Franson interference with a phase of $\phi_{\text {Franson }}=\phi_{A}+\phi_{B}$. All of our measurements in the superposition or Franson basis are performed at maximal Franson interference contrast $\left(\phi_{\text {Franson }}=0\right.$ or $\left.\pi\right)$, which requires phase stability of the PMZIs over the measurement time. Active phase stabilization of both PMZIs is achieved by a control loop of a piezoactuator displacing an interferometer mirror and the difference signal from two photodiodes indicating the interference contrast. This interference signal is provided by a $780.241-\mathrm{nm}$ stabilization laser (Toptica DL Pro) propagating in the same spatial interferometer mode as the single photons. It is injected into the PMZIs via the unused port of the first polarizing beam splitter (PBS) and measured at the output of the unused port of the second PBS, where the polarization contrast is measured by fast photodiodes (Thorlabs-DET $10 \mathrm{~A} / \mathrm{M}$ ) in a polarization basis conjugate to the polarization basis defined by the PMZIs. The stabilization laser is frequency locked to a hyperfine transition of ${ }^{85} \mathrm{Rb}$, obtained by saturated absorption spectroscopy, resulting in a wavelength stability of $\sim 0.6 \mathrm{fm} / \mathrm{min}$.

We choose the measurement basis in the energy-time domain by changing the polarization measurement basis after the PMZI, effectively switching the interferometer on or off by erasing or revealing the interferometer path information. Performing a polarization measurement in the PMZI-defined rectilinear basis corresponds to a measurement in the computational basis, while projecting the photons in a mutually unbiased polarization basis corresponds to a measurement in the Franson basis (see Supplemental Material, Sec. III [47] for a stringent formal treatment). Noise is optically added to the measurement data by means of fine-adjustable light emitting diodes (LEDs) powered by a battery, ensuring time-invariant noise generation. We detect both polarization components on Alice's (detectors A0 and A1) and Bob's (detectors B0 and B1) side by means of multimode-coupled singlephoton avalanche diodes (Excelitas SPCM-800-11) with a measured FWHM timing jitter of $<800$ ps between two detectors. The detection events are time tagged employing a time-to-amplitude converter (AIT TTM8000) with a clock resolution of 82.3 ps.

Postprocessing of the time-tagged data is realized by binning the detection events of each channel into dimension-dependent time bins of duration $t_{d}=F / d$, where $F$ is the duration of one frame. Since the imbalance of our interferometers is fixed and corresponds to 32 clock cycles, only time-bin durations which obey $f t_{d}=32$ clock cycles give rise to well-defined Franson interference, $|i, i\rangle+e^{i \phi_{\text {Franson }}}|i+f, i+f\rangle$, where $f$ is an integer corresponding to the time-bin shift. To this end, in order to see interference, we investigate dimensions which satisfy $d=[(f F) / 32]$ for integer $f$ and $d$. For our setup parameters and for a time-frame duration of $F=320$ clock cycles this corresponds to $d \in\{10,20,40,80\}$.

The discretizations to different dimensions are performed on the same set of measurement data. Since we are tracking photons emitted from a photon pair source, our state space is intrinsically bipartite, and only those time frames which contain exactly one detection event on Alice's side and exactly one on Bob's side are kept; all others are discarded (e.g., no detection event in Alice's and 1 detection event in Bob's detectors per frame). The detection events which are kept are then sorted into count matrices pertaining to the detectors that clicked (A0-B0, A1-B1, A0-B1, A1-B0). These four matrices in both measurement bases are used to reconstruct the part of the state $\rho_{\mathrm{ET}}$ required in the subsequent entanglement certification.

Since the timing jitter of the detectors is 1 order of magnitude greater than the clock resolution of the time tagger, our overall timing resolution is dominated by the detector jitter. Therefore, cross-talk errors between time bins will sharply increase once the time-bin duration $t_{d}$ is on the order of the timing jitter of the detector, which is the case for $t_{d}=8$ clock cycles $=658.4$ ns.

The witness used to certify entanglement was derived using the entropy vector formalism in Ref. [65]. For each dimension $d$, the underlying state $\rho_{\mathrm{ET}}$ is not separable (i.e., entanglement is certified) if $W\left(\rho_{\mathrm{ET}}, d\right)>0$, where

$$
\begin{aligned}
& W\left(\rho_{\mathrm{ET}}, d\right) \\
& \quad:=\sum_{i}^{d-f}\left|\left\langle i i\left|\rho_{\mathrm{ET}}\right| i+f, i+f\right\rangle\right| \\
& \quad-\sqrt{\left\langle i, i+f\left|\rho_{\mathrm{ET}}\right| i, i+f\right\rangle\left\langle i+f, i\left|\rho_{\mathrm{ET}}\right| i+f, i\right\rangle} .
\end{aligned}
$$

In order to compute the witness, one must reconstruct the underlying density matrix elements of $\rho_{\mathrm{ET}}$ from the 
experimental count matrices. These also depend on the polarization d.o.f., due to the use of a postselection-free Franson interferometer. Details of how to compute the witness from the count matrices produced by the experiment can be found in the Supplemental Material, Sec. I [47].

\section{APPENDIX B: OAM ENTANGLEMENT EXPERIMENT}

We generate pairs of photons entangled in the orbitalangular-momentum d.o.f. by pumping a 5-mm-long ppKTP crystal quasi-phase-matched for type-II SPDC. We use a 405-nm diode laser (Toptica iBeam Smart 405 HP) that is coupled to a single-mode optical fiber to ensure the best possible transverse coherence and mode profile, which is essential to obtain high-dimensionally entangled pairs of photons. The UV beam is focused by a $500-\mathrm{mm}$ lens to a spot size of $430 \mu \mathrm{m}\left(1 / e^{2}\right.$ beam diamater $)$ at the ppKTP crytal. We similarly temperature tune this crystal to produce pairs of wavelength-degenerate, orthogonally polarized photons at $810 \mathrm{~nm}$. The photon pairs are recollimated by a $300-\mathrm{mm}$ lens. This time the polarization d.o.f. of the photons is solely used to deterministically split the photons at a polarization beam splitter, such that their spatial mode can be measured independently of each other. The photons are then made incident on phase-only spatial light modulators (Holoeye PLUTO), where a combination of computer-generated holograms and single-mode fibers are used to perform a generalized projective measurement in the OAM state space. Finally, the photons are detected by avalanche photodiodes and coincidence measurements are recorded within a coincidence time window of $5 \mathrm{~ns}$ using a custom-built logic. In the computational basis, measurements of photonic OAM may be accomplished by displaying a hologram generating the opposite OAM value, thus resulting in an outgoing beam with a flat wavefront with an OAM value of $\ell=0$ that will couple efficiently to the SMF using a $10 \times$ microscope objective. This technique is also known as phase flattening and has been widely used to measure the OAM content of an unknown beam [66]. However, in order to certify entanglement, it is necessary to perform measurements in additional bases besides the computational (OAM) basis, which leads to more complex mode structures (see Supplemental Material, Sec. V [47]). Thus, a more elaborate measurement scheme is required to accurately measure the general OAM state of the experimentally generated entangled pairs. We use a recently introduced technique called intensity flattening [55], that allows one to measure any arbitrary transverse spatial mode of light, including modes in any mutually unbiased basis of OAM. Although lossy, this technique yields extremely high detection fidelities. Using this source, after taking into account the lossy intensity masking holograms implemented at the spatial light modulators, we achieve coincidence count rates of $500 \mathrm{~Hz}$ in the fundamental Gaussian mode, $1000 \mathrm{~Hz}$ in the first-order OAM modes $(\ell= \pm 1), 700 \mathrm{~Hz}$ in the second-order OAM modes $(\ell= \pm 2)$, and $400 \mathrm{~Hz}$ in the third-order OAM modes $(\ell= \pm 3)$. The associated singles count rates are given by $13,20,15$, and $11 \mathrm{kHz}$.

[1] N. Friis, G. Vitagliano, M. Malik, and M. Huber, Entanglement Certification from Theory to Experiment, Nat. Rev. Phys. 1, 72 (2019).

[2] A. Acín, N. Brunner, N. Gisin, S. Massar, S. Pironio, and V. Scarani, Device-Independent Security of Quantum Cryptography against Collective Attacks, Phys. Rev. Lett. 98, 230501 (2007).

[3] D. Salart, A. Baas, C. Branciard, N. Gisin, and H. Zbinden, Testing the Speed of 'Spooky Action at a Distance,' Nature (London) 454, 861 (2008).

[4] T. Inagaki, N. Matsuda, O. Tadanaga, M. Asobe, and H. Takesue, Entanglement Distribution over $300 \mathrm{~km}$ ofFfiber, Opt. Express 21, 23241 (2013).

[5] S. Wengerowsky, S. K. Joshi, F. Steinlechner, J. R. Zichi, S. M. Dobrovolskiy, R. van der Molen, J. W. N. Los, V. Zwiller, M. A. M. Versteegh, A. Mura, D. Calonico, M. Inguscio, H. Hübel, L. Bo, T. Scheidl, A. Zeilinger, A. Xuereb, and R. Ursin, Entanglement Distribution over a 96-km-Long Submarine Optical Fiber, Proc. Natl. Acad. Sci. U.S.A. 116, 6684 (2019).

[6] T. Scheidl, R. Ursin, A. Fedrizzi, S. Ramelow, X.-S. Ma, T. Herbst, R. Prevedel, L. Ratschbacher, J. Kofler, T. Jennewein, and A. Zeilinger, Feasibility of $300 \mathrm{~km}$ Quantum Key Distribution with Entangled States, New J. Phys. 11, 085002 (2009).

[7] M. Krenn, J. Handsteiner, M. Fink, R. Fickler, and A. Zeilinger, Twisted Photon Entanglement through Turbulent Air across Vienna, Proc. Natl. Acad. Sci. U.S.A. 112, 14197 (2015).

[8] J. Yin et al., Satellite-Based Entanglement Distribution over 1200 Kilometers, Science 356, 1140 (2017).

[9] F. Steinlechner, S. Ecker, M. Fink, B. Liu, J. Bavaresco, M. Huber, T. Scheidl, and R. Ursin, Distribution of HighDimensional Entanglement via an Intra-City Free-Space Link, Nat. Commun. 8, 15971 (2017).

[10] T. Ikuta and H. Takesue, Four-Dimensional Entanglement Distribution over 100 km, Sci. Rep. 8, 817 (2018).

[11] D. Cozzolino, D. Bacco, B. Da Lio, K. Ingerslev, Y. Ding, K. Dalgaard, P. Kristensen, M. Galili, K. Rottwitt, S. Ramachandran, and L. K. Oxenløwe, Orbital Angular Momentum States Enabling Fiber-Based High-Dimensional Quantum Communication, Phys. Rev. Applied 11, 064058 (2019).

[12] J. Liu, I. Nape, Q. Wang, A. Valles, J. Wang, and A. Forbes, Multi-Dimensional Entanglement Transport through Single-Mode Fibre, arXiv:1904.03114.

[13] D. Cozzolino, E. Polino, M. Valeri, G. Carvacho, D. Bacco, N. Spagnolo, L. K. K. Oxenløwe, and F. Sciarrino, Air-Core Fiber Distribution of Hybrid Vector Vortex-Polarization Entangled States, Adv. Opt. Photonics 1, 1 (2019).

[14] H. Cao, S.-C. Gao, C. Zhang, J. Wang, D.-Y. He, B.-H. Liu, Z.-W. Zhou, G.-X. Zhu, Y.-J. Chen, Z.-H. Li, S.-Y. Yu, Y.-F. Huang, C.-F. Li, and G.-C. Guo, Distribution of HighDimensional Orbital Angular Momentum Entanglement at 
Telecom Wavelength over $1 \mathrm{~km}$ OAM Fiber, arXiv:1811 .12195 .

[15] R. T. Thew, A. Acín, H. Zbinden, and N. Gisin, Bell-Type Test of Energy-Time Entangled Qutrits, Phys. Rev. Lett. 93, 010503 (2004).

[16] J. Brendel, N. Gisin, W. Tittel, and H. Zbinden, Pulsed Energy-Time Entangled Twin-Photon Source for Quantum Communication, Phys. Rev. Lett. 82, 2594 (1999).

[17] A. K. Jha, M. Malik, and R. W. Boyd, Exploring EnergyTime Entanglement Using Geometric Phase, Phys. Rev. Lett. 101, 180405 (2008).

[18] J.-P. W. MacLean, J. M. Donohue, and K. J. Resch, Direct Characterization of Ultrafast Energy-Time Entangled Photon Pairs, Phys. Rev. Lett. 120, 053601 (2018).

[19] A. Vaziri, G. Weihs, and A. Zeilinger, Experimental TwoPhoton, Three-Dimensional Entanglement for Quantum Communication, Phys. Rev. Lett. 89, 240401 (2002).

[20] J. Leach, B. Jack, J. Romero, A. K. Jha, A. M. Yao, S. Franke-Arnold, D. G. Ireland, R. W. Boyd, S. M. Barnett, and M. J. Padgett, Quantum Correlations in Optical AngleOrbital Angular Momentum Variables, Science 329, 662 (2010).

[21] M. Krenn, M. Malik, M. Erhard, and A. Zeilinger, Orbital Angular Momentum of Photons and the Entanglement of Laguerre-Gaussian Modes, Phil. Trans. R. Soc. A 375 (2017).

[22] M. Erhard, R. Fickler, M. Krenn, and A. Zeilinger, Twisted Photons: New Quantum Perspectives in High Dimensions, Light Sci. Appl. 7, 17146 (2018).

[23] J. C. Howell, R. S. Bennink, S. J. Bentley, and R. W. Boyd, Realization of the Einstein-Podolsky-Rosen Paradox Using Momentum- and Position-Entangled Photons from Spontaneous Parametric Down Conversion, Phys. Rev. Lett. 92, 210403 (2004).

[24] C. Schaeff, R. Polster, M. Huber, S. Ramelow, and A. Zeilinger, Experimental Access to Higher-Dimensional Entangled Quantum Systems Using Integrated Optics, Optica 2, 523 (2015).

[25] J. Wang, S. Paesani, Y. Ding, R. Santagati, P. Skrzypczyk, A. Salavrakos, J. Tura, R. Augusiak, L. Mančinska, D. Bacco, D. Bonneau, J. W. Silverstone, Q. Gong, A. Acín, K. Rottwitt, L. K. Oxenløwe, J. L. O'Brien, A. Laing, and M. G. Thompson, Multidimensional Quantum Entanglement with Large-Scale Integrated Optics, Science 360, 285 (2018).

[26] T. Kraft, C. Ritz, N. Brunner, M. Huber, and O. Gühne, Characterizing Genuine Multilevel Entanglement, Phys. Rev. Lett. 120, 060502 (2018).

[27] M. Huber and M. Pawłowski, Weak Randomness in DeviceIndependent Quantum Key Distribution and the Advantage of Using High-Dimensional Entanglement, Phys. Rev. A 88, 032309 (2013).

[28] H. Bechmann-Pasquinucci and A. Peres, Quantum Cryptography with 3-State Systems, Phys. Rev. Lett. 85, 3313 (2000).

[29] H. Bechmann-Pasquinucci and W. Tittel, Quantum Cryptography Using Larger Alphabets, Phys. Rev. A 61, 062308 (2000).

[30] N. J. Cerf, M. Bourennane, A. Karlsson, and N. Gisin, Security of Quantum Key Distribution Using d-Level Systems, Phys. Rev. Lett. 88, 127902 (2002).
[31] G. M. Nikolopoulos and G. Alber, Security Bound of Two-Basis Quantum-Key-Distribution Protocols Using Qudits, Phys. Rev. A 72, 032320 (2005).

[32] L. Sheridan and V. Scarani, Security Prooffor Quantum Key Distribution Using Qudit Systems, Phys. Rev. A 82, 030301 (R) (2010).

[33] I. Ali-Khan, C. J. Broadbent, and J. C. Howell, LargeAlphabet Quantum Key Distribution Using Energy-Time Entangled Bipartite States, Phys. Rev. Lett. 98, 060503 (2007).

[34] Z. Zhang, M. Tengner, T. Zhong, F. N. C. Wong, and J. H. Shapiro, Entanglement's Benefit Survives an EntanglementBreaking Channel, Phys. Rev. Lett. 111, 010501 (2013).

[35] T. Zhong, H. Zhou, R. D. Horansky, C. Lee, V. B. Verma, A. E. Lita, A. Restelli, J. C. Bienfang, R. P. Mirin, T. Gerrits, S. W. Nam, F. Marsili, M. D. Shaw, Z. Zhang, L. Wang, D. Englund, G. W. Wornell, J. H. Shapiro, and F. N. C. Wong, Photon-Efficient Quantum Key Distribution Using Time-Energy Entanglement with High-Dimensional Encoding, New J. Phys. 17, 022002 (2015).

[36] A. Sit, F. Bouchard, R. Fickler, J. Gagnon-Bischoff, H. Larocque, K. Heshami, D. Elser, C. Peuntinger, K. Günthner, B. Heim, C. Marquardt, G. Leuchs, R. W. Boyd, and E. Karimi, High-Dimensional Intracity Quantum Cryptography with Structured Photons, Optica 4, 1006 (2017).

[37] F. Bouchard, K. Heshami, D. England, R. Fickler, R. W. Boyd, B.-G. Englert, L. L. Sánchez-Soto, and E. Karimi, Experimental Investigation of High-Dimensional Quantum Key Distribution Protocols with Twisted Photons, Quantum 2, 111 (2018).

[38] F. Zhu, M. Tyler, N. H. Valencia, M. Malik, and J. Leach, Are High-Dimensional Entangled States Robust to Noise?, arXiv:1908.08943.

[39] D. Collins, N. Gisin, N. Linden, S. Massar, and S. Popescu, Bell Inequalities for Arbitrarily High-Dimensional Systems, Phys. Rev. Lett. 88, 040404 (2002).

[40] A. Peres, Separability Criterion for Density Matrices, Phys. Rev. Lett. 77, 1413 (1996).

[41] M. Horodecki, P. Horodecki, and R. Horodecki, Separability of Mixed States: Necessary and Sufficient Conditions, Phys. Lett. A 223, 1 (1996).

[42] G. Vidal and R. Tarrach, Robustness of Entanglement, Phys. Rev. A 59, 141 (1999).

[43] M. Horodecki and P. Horodecki, Reduction Criterion of Separability and Limits for a Class of Distillation Protocols, Phys. Rev. A 59, 4206 (1999).

[44] L. Lami and M. Huber, Bipartite Depolarizing Maps, J. Math. Phys. (N.Y.) 57, 092201 (2016).

[45] J. Bavaresco, N. H. Valencia, C. Klöckl, M. Pivoluska, P. Erker, N. Friis, M. Malik, and M. Huber, Measurements in Two Bases Are Sufficient for Certifying High-Dimensional Entanglement, Nat. Phys. 14, 1032 (2018).

[46] T. Brougham and S. M. Barnett, Information Communicated by Entangled Photon Pairs, Phys. Rev. A 85, 032322 (2012).

[47] See Supplemental Material at http://link.aps.org/ supplemental/10.1103/PhysRevX.9.041042 for the derivation of an entanglement witness, the definition of the noise fraction (NF), the discretization of the time domain, details on the error analysis, and correlation measurements of the OAM experiment, which includes Refs. [48]. 
[48] M. Huber, M. Perarnau-Llobet, and J. I. de Vicente, Entropy Vector Formalism and the Structure of Multidimensional Entanglement in Multipartite Systems, Phys. Rev. A 88, 042328 (2013).

[49] J. D. Franson, Bell Inequality for Position and Time, Phys. Rev. Lett. 62, 2205 (1989).

[50] D. V. Strekalov, T. B. Pittman, A. V. Sergienko, Y. H. Shih, and P. G. Kwiat, Postselection-Free Energy-Time Entanglement, Phys. Rev. A 54, R1 (1996).

[51] P. G. Kwiat, Hyper-Entangled States, J. Mod. Opt. 44, 2173 (1997).

[52] J. T. Barreiro, N. K. Langford, N. A. Peters, and P. G. Kwiat, Generation of Hyperentangled Photon Pairs, Phys. Rev. Lett. 95, 260501 (2005).

[53] T. Kim, M. Fiorentino, and F. N. C. Wong, Phase-Stable Source of Polarization-Entangled Photons Using a Polarization Sagnac Interferometer, Phys. Rev. A 73, 012316 (2006).

[54] A. Fedrizzi, T. Herbst, A. Poppe, T. Jennewein, and A. Zeilinger, A Wavelength-Tunable Fiber-Coupled Source of Narrowband Entangled Photons, Opt. Express 15, 15377 (2007).

[55] F. Bouchard, N. H. Valencia, F. Brandt, R. Fickler, M. Huber, and M. Malik, Measuring Azimuthal and Radial Modes of Photons, Opt. Express 26, 31925 (2018).

[56] T. Durt, B.-G. Englert, I. Bengtsson, and K. Życzkowski, On Mutually Unbiased Bases, Int. J. Quantum. Inform. 08, 535 (2010).

[57] C. Spengler, M. Huber, S. Brierley, T. Adaktylos, and B. C. Hiesmayr, Entanglement Detection via Mutually Unbiased Bases, Phys. Rev. A 86, 022311 (2012).
[58] J. Svozilík, J. Peŕina, Jr., and J. P. Torres, High Spatial Entanglement via Chirped Quasi-Phase-Matched Optical Parametric Down-Conversion, Phys. Rev. A 86, 052318 (2012).

[59] M. P. Peloso, I. Gerhardt, C. Ho, A. Lamas-Linares, and C. Kurtsiefer, Daylight Operation of a Free Space, Entanglement-Based Quantum Key Distribution System, New J. Phys. 11, 045007 (2009).

[60] S.-K. Liao et al., Long-Distance Free-Space Quantum Key Distribution in Daylight towards Inter-Satellite Communication, Nat. Photonics 11, 509 (2017).

[61] M. Krenn, J. Handsteiner, M. Fink, R. Fickler, R. Ursin, M. Malik, and A. Zeilinger, Twisted Light Transmission over 143 km, Proc. Natl. Acad. Sci. U.S.A. 113, 13648 (2016).

[62] M. Mirhosseini, M. Malik, Z. Shi, and R. W. Boyd, Efficient Separation of the Orbital Angular Momentum Eigenstates of Light, Nat. Commun. 4, 2781 (2013).

[63] S. Bäuml, A. Winter, and D. Yang, Every Entangled State Provides an Advantage in Classical Communication, J. Math. Phys. (N.Y.) 60, 072201 (2019).

[64] M. Piani and J. Watrous, All Entangled States Are Useful for Channel Discrimination, Phys. Rev. Lett. 102, 250501 (2009).

[65] M. Huber and J. I. de Vicente, Structure of Multidimensional Entanglement in Multipartite Systems, Phys. Rev. Lett. 110, 030501 (2013).

[66] A. Mair, A. Vaziri, G. Weihs, and A. Zeilinger, Entanglement of the Orbital Angular Momentum States of Photons, Nature (London) 412, 313 (2001). 